\title{
Personalised pulmonary rehabilitation in COPD
}

\author{
Emiel F.M. Wouters ${ }^{1,2}$, Birgit B.R.E.F. Wouters ${ }^{3}$, Ingrid M.L. Augustin², \\ Sarah Houben-Wilke ${ }^{2}$, Lowie E.G.W. Vanfleteren ${ }^{1}$ and Frits M.E. Franssen ${ }^{1,2}$ \\ Number 5 in the Series "Personalised medicine in respiratory diseases" \\ Edited by Renaud Louis and Nicolas Roche
}

Affiliations: ${ }^{1}$ Maastricht University Medical Center, Dept of Respiratory Diseases, Maastricht, The Netherlands. ${ }^{2} \mathrm{CIRO}+$, Centre of Expertise for Chronic Organ Failure, Horn, The Netherlands. ${ }^{3}$ Dept of Health, Ethics and Society, CAPHRI School for Public Health and Primary Care, Faculty of Health Medicine and Life Science, Maastricht University Medical Center, Maastricht, The Netherlands.

Correspondence: Emiel F.M. Wouters, Maastricht University Medical Center, Dept Respiratory Diseases, P.O. Box 5800, 6202 AZ Maastricht, The Netherlands. E-mail: e.woutersamumc.nl

@ERSpublications

Pulmonary rehabilitation offers an integrated approach for personalised management of patients with COPD http://ow.ly/Hyog30irp1h

Cite this article as: Wouters EFM, Wouters BBREF, Augustin IML, et al. Personalised pulmonary rehabilitation in COPD. Eur Respir Rev 2018; 27: 170125 [https://doi.org/10.1183/16000617.0125-2017].

ABSTRACT This review summarises ongoing developments in personalised medicine and individualised medicine in chronic obstructive pulmonary disease (COPD). Currently applied classification systems largely ignore the complexity and heterogeneity of the COPD syndrome. Personalised medicine has to consider the influence of unique circumstances of the person, which contribute to this heterogeneity and complexity. Pulmonary rehabilitation is described as a comprehensive, individualised intervention based on thorough assessment of identifiable treatable traits. Partnership in care will become a crucial factor to improve and maintain health. Tolerating uncertainty and unpredictability will enrich future doctor-patient relationships.

\section{Introduction}

Chronic obstructive pulmonary disease (COPD) is among the five leading causes of death in the developed world and is a rapidly rising cause of death in most other countries [1]. COPD is defined as a common, preventable and treatable disease characterised by persistent respiratory symptoms and chronic airflow limitation due to airway and/or alveolar abnormalities, usually caused by significant exposure to noxious particles or gases [2]. Cigarette smoking is clearly the predominant cause of COPD development, yet other environmental factors such as exposure to biomass fuels and air pollution may contribute [3]. Besides these exposures, it is assumed that host factors predispose individuals to develop COPD [2].

Previous articles in this series: No. 1: Chung KF. Personalised medicine in asthma: time for action. Eur Respir Rev 2017; 26: 170064. No. 2: Bonsignore MR, Suarez Giron MC, Marrone O, et al. Personalised medicine in sleep respiratory disorders: focus on obstructive sleep apnoea diagnosis and treatment. Eur Respir Rev 2017; 26: 170069. No. 3: Mascaux C, Tomasini P, Greillier L, et al. Personalised medicine for nonsmall cell lung cancer. Eur Respir Rev 2017; 26: 170066. No. 4: Noell G, Faner R, Agusti A. From systems biology to P4 medicine: applications in respiratory medicine. Eur Respir Rev 2018; 27: 170110.

Received: Nov 152017 | Accepted after revision: Feb 122018

Provenance: Commissioned article, peer reviewed.

Copyright OERS 2018. ERR articles are open access and distributed under the terms of the Creative Commons Attribution Non-Commercial Licence 4.0. 
Despite the commendable effort of a focus on respiratory symptoms and the addition of alveolar abnormalities in its most recent revision, the Global Initiative for Chronic Obstructive Lung Disease definition of COPD largely approaches it as a single disease entity, operationally defined by abnormal spirometry and more specifically by the postbronchodilator forced expiratory volume in $1 \mathrm{~s}(\mathrm{FEV} 1) /$ forced vital capacity ratio. Based on the observational studies of Fletcher and PETO [4], the natural history of COPD has been viewed as synonymous with progressive decline in FEV1 from early adulthood and throughout life.

This airflow limitation centred approach of COPD as a mainly respiratory disease largely ignores our increasing understanding of COPD as a complex and heterogeneous condition with a variety of intra- and extrapulmonary components [5-7]. The term "heterogeneous" indicates that not all of these components are present in all patients at any given time, while "complex" reflects the dynamic, nonlinear interactions over time between these components [8].

Pulmonary rehabilitation aims to stabilise or reverse the physiopathological and psychopathological manifestations of the disease and attempts to bring the patient to the highest possible functional capacity [9]. This review positions pulmonary rehabilitation as an integrated personalised management strategy in partnership with the patient.

\section{Current COPD approach: black-and-white reductionism}

Current COPD guidelines reflect a reductionist thinking and the current classification system, based on airflow limitation, dyspnoea sensation and risk of exacerbations, tries to break down ambiguity to achieve more certainty and agreement [2]. This approach is valorised by linking such classification to so-called state-of-the-art, evidence-based prescription advice for a variety of medications [2]. Indeed, our learnt behaviour as healthcare professionals is to move towards a comfort zone of high agreement and high certainty [10]. Newton's clockwork universe, in which big problems can be broken down into smaller ones, analysed and solved by rational deduction, has strongly influenced our current practice of medicine [10]. Our approach to COPD as a single disease, operationally defined by results of spirometry, is an excellent example of this machine metaphor: doctors have to tackle this obstruction using the knowledge and skills they have acquired. In addition, the classification of COPD into ABCD groups is a model of transformation of patient's greyscale narrative into a black-and-white diagnosis than can be categorised and labelled, as severity and risk assessment is based on self-reported symptoms and experiences [2, 11]. Current medicine largely neglects Osler's statement that medicine is a science of uncertainty and an art of probability [12].

Patients with COPD experience important limitations in daily life, often compelling them to rely on external help $[13,14]$. Patients have to cope with their chronic condition and they develop extensive experience in the daily management of the disease [15-17]. Doctors' disease management goals poorly address the residual disability as characteristic of chronic conditions and poorly focus on improvement of functionality and independence, improvement in adaptability and flexibility and creation of better control, autonomy and self-management for patients with COPD [18, 19]. This approach fits with the concept of health as the ability to adapt and to self-manage, in the face of physical, social and emotional challenges [20].

Current COPD management is still based on predictable and binary outcomes, supported by randomised clinical trials, does not tolerate uncertainty and is far from personalised management algorithms maximising health benefits with respect to symptoms for the individual patient.

\section{The many faces of personalised medicine}

The definition and scope of the term personalised medicine vary widely and the terms precision medicine, personalised and individualised medicine are often used interchangeably. Some of these definitions are summarised in table 1. The term "personalised medicine" has been used for many years, but has engendered considerable confusion by the hackneyed use of the term "personalised" in daily life and practice. "Person" refers to a human being and medicine in his broadest sense can be defined as the science and practice of diagnosis, treatment and prevention of disease [21,31]. In a personalised medicine approach, human beings can be considered as a complex adaptive system, in which a collection of individual agents act in ways that are not always predictable and whose actions are interconnected so that the action of one part changes the context of other agents [10]. Human health and illness are the consequence of all these dynamic interactions. Health can therefore only be maintained though a holistic approach that accepts unpredictability and builds on subtle, emergent forces within the overall system [32]. In this definition of personalised medicine, holistic tailoring of the decisions and practices to the individual patient is considered very important. 


\section{TABLE 1 Definitions of personalised medicine}

\section{Personalised} medicine

P4 medicine

Precision medicine

Individualised medicine
Human health and illness are the consequence of all dynamic interactions within human beings, considered as complex adaptive systems [10]

A medical model that proposes the customisation of healthcare, with decisions and practices being tailored to the individual patient by use of genetic or other information [21]

A form of medicine that uses information about a person's genes, proteins and environment to prevent, diagnose and treat disease [22]

Tailoring of medical treatment to the specific characteristics of each patient by classifying individuals into subpopulations that are uniquely or disproportionately susceptible to a particular disease or responsive to a specific treatment [23]

The right treatment for the right patient at the right time $[24,25]$

Medicine that is predictive, preventive, personalised and participatory to optimise wellness and minimise disease for each individual [26]

Treatments targeted to the needs of individual patients on the basis of genetic, biomarker, phenotypic or psychosocial characteristics that distinguish a given patient from other patients with similar clinical presentations [27-29]

Relates not only to medicine, but also focuses on the flow of biological data and relevant medical information directly to individuals as drivers of healthcare [30]

The convergence of systems biology, the digital revolution and consumer-driven healthcare has introduced the concept of $\mathrm{P} 4$ medicine (medicine that is predictive, preventive, personalised and participatory) to optimise wellness and minimise disease for each individual [26]. Personalised in this definition refers to the uniqueness of individuals in terms of genetics, while participatory refers to the shift of individuals from passive recipients of expert advice, characteristic of the predigital medicine, to activated and networked consumers: collectively, patients and consumers constitute a vital stakeholder group in P4 medicine [26].

The term "precision medicine" has been proposed instead of personalised medicine [27-29]. Precision medicine is defined as "treatments targeted to the needs of individual patients on the basis of genetic, biomarker, phenotypic or psychosocial characteristics that distinguish a given patient from other patients with similar clinical presentations. The final objective of precision medicine is to tailor medical treatment to improve clinical outcomes for individual patients while minimising side-effects for those likely to respond to a given treatment" [27]. "Stratification" in this definition refers to the division of patients with a certain disease into subgroups, based on a characteristic of some sort, who respond more frequently to a particular drug, or, alternatively, are at decreased risk of side-effects in response to a certain treatment. This definition fits with the approach upheld in the European Union that precision medicine must provide the right treatment, for the right patient, at the right dose and at the right time [24]. The current convergence of genetics, informatics, imaging, cell sorting, epigenetics, proteomics and metabolomics is rapidly expanding the scope of precision medicine by refining the classification of diseases with important prognostic and treatment implications. Precision medicine has already contributed importantly to a high level of biological precision in intervening identified aberrant pathways in different disease conditions [27]. Crucial will be the requirement that information is derived from the individual [30]. The possibilities to uniquely tailor treatment to the individual are unimaginable.

The term "individualised medicine" has been suggested because it relates not only to medicine that is particularised to a human being, the definition of an individual, but also to the future impact of digital technologies on individuals driving their healthcare [30]. Indeed, there will be an increasing flow of biological data and relevant medical information directly to the individual in order to maintain and improve the individual's health [30]. Crucial for individualised medicine is digitising the human being. Multiple layers of data can now be obtained for any individual. These layers could include demographics and social data, biosensors to capture the individual's physiome, imaging to depict the anatomy and the biology of the various omics technologies: genome-DNA sequencing, transcriptomics, proteomics, metabolomics, microbiomics and epigenomics. To denote the multiple biologic omic technologies the term "panoromic" has been suggested for portraying the concept of big biological data [30]. Fundamental to individualised medicine is the ability to analyse these immense datasets and to extract all of the useful, salient information. Crucial in individualised medicine will be the ability to cover the lifespan of the individual "from pre-womb to tomb" [30]. These opportunities of individualised medicine will constitute an enormous challenge to the medical community and the current practicing role of medical doctors. 
Algorithms, artificial intelligence and machine learning are some of the crucial steps to reboot the way healthcare can be rendered [30].

Individuals are not only distinguished by their biological variability; they differ greatly in terms of how disease affects their lives. People have different personalities, resilience and resources that influence how they adapt to illness. Diseases do not just affect individuals: they affect their families and friends and their communities. Understanding these unique life circumstances that influence disease susceptibility, phenotype and response to treatment is critical to patient care. Personomics, integrating psychological, social, cultural, behavioural and economic factors is as crucial for a personalised management approach as other panoromic methodologies [33].

\section{Stratified medicine as a path towards personalised medicine in COPD}

In order to describe the complexity and heterogeneity of COPD, the identification and grouping of key elements of the COPD syndrome into clinically meaningful and useful subgroups or phenotypes that can guide therapy more effectively can be considered. The classical definition of a phenotype reflects the observable structural and functional characteristics of an organism determined by its genotype and modulated by its environment [34]. Concerning COPD phenotypes, it is proposed that a phenotype must be able to classify patients into specific subgroups that provide prognostic information and enable better determination of appropriate therapy that alters clinically meaningful outcomes. The ultimate goal of phenotyping in medicine is to allow the identification of patient groups with unique prognostic or therapeutic perspectives [34]. With progress in knowledge and developments in physiology, lung imaging, medical biology and genetics, the identification of phenotypes of COPD is an urgent medical need. Sampling different populations in the COPD disease spectrum including asymptomatic smokers and age-matched nonsmoking controls to mirror the population of COPD patients and to characterise disease-specific traits must be realised and longitudinal studies of patients with COPD must validate the usefulness of the established phenotypes and must explore sources of variability in phenotypes and the temporal nature $[35,36]$.

The appellation "treatable traits" has recently been introduced [8]. It refers to a label-free, precision medicine approach to the diagnosis and management of chronic airway diseases in general. Management should be based on individual treatable traits and these treatable traits are summarised into three groups: pulmonary treatable traits, extrapulmonary treatable traits and treatable behavioural and lifestyle risk factors. The patient's environment has been included as a distinct treatable trait, underlining the increasing awareness and importance of personomics in an integrated management strategy [8]. Still, this approach focuses on the assessment of several, separate traits, while integrated, holistic approaches are needed to tackle the complexity of every unique patient. Furthermore, it must be realised that addressing a treatable trait must be based on profound understanding of underlying, individual factors. For example, a variety of social and personal barriers and enablers can limit or encourage participation in physical activity in patients with COPD (figure 1) [37]. In order to address physical activity as treatable trait, it will be important to understand these patient-specific cognitive influences on participation in physical activity, to facilitate individually tailored treatment planning [37].

\section{Pulmonary rehabilitation: personalised management of COPD}

Personalised and patient-centred approaches for patients with COPD are not new in pulmonary medicine. Indeed, the first authoritative statement of pulmonary rehabilitation introduced pulmonary rehabilitation as an art of medical practice, wherein an individually tailored, multidisciplinary programme is formulated through accurate diagnosis, therapy, emotional support and education to stabilise or reverse both physiopathological and psychopathological manifestations of pulmonary diseases. Such a programme must attempt to return the patient to the highest possible functional capacity allowed by the handicap and overall life situation [9]. In 1994, the National Institutes of Health defined pulmonary rehabilitation as a multidimensional continuum of services for the patient and the family supplied by an integrated team of specialists [38]. More recently, pulmonary rehabilitation has been defined as a comprehensive intervention based on a "thorough" patient assessment followed by patient-tailored therapies designed to improve the physical and psychological condition of patients with chronic respiratory disease and to promote the long-term adherence to health-enhancing behaviours [39]. Offering a patient-tailored, individualised, comprehensive intervention targeting complex needs to improve physiological, psychological and social outcomes and to promote long-term adherence to health-enhancing behaviours must be the cornerstone of every rehabilitation programme [38-40]. Unfortunately, this integrated, comprehensive and personalised management approach is eroded in many programmes to exercise training with or without basic self-management instructions. To offer a holistic approach, pulmonary rehabilitation programmes must be organised around the customer and the identified needs and traits. By applying sociotechnical principles, modular patient-tailored interventions can be implemented and organised as integrated practice units 


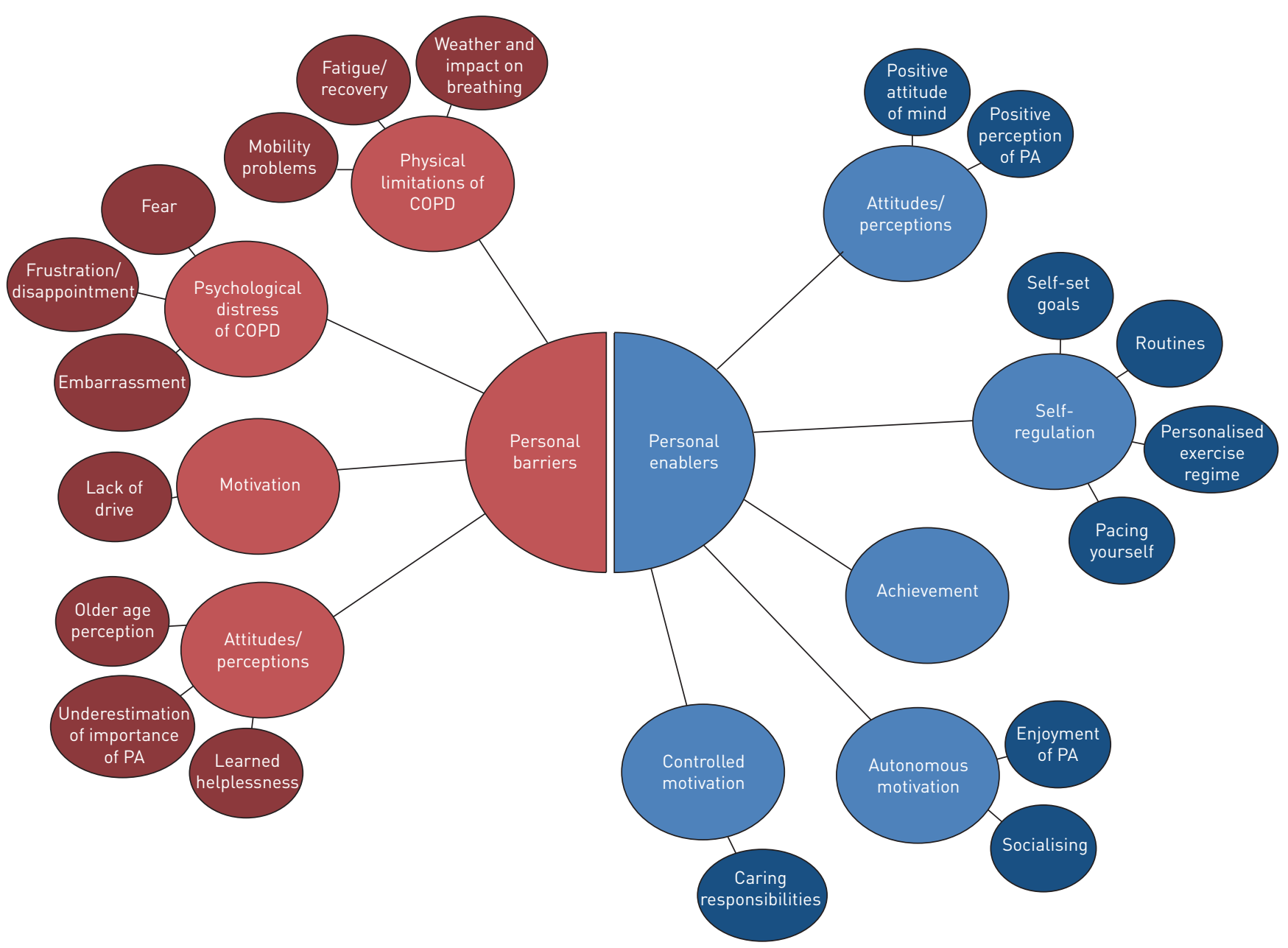

FIGURE 1 Examples of personal barriers and enablers that both encourage and limit participation in physical activity for patients with chronic obstructive pulmonary disease (COPD). PA: physical activity. Reproduced from [37] with permission.

(figure 2) [41]. Crucial to the identification of realised values for the patient is the identification of responses based on a multidimensional index. It has been reported that outcomes by such personalised programme are nonlinear and really can downsize the burden of disease for the individual patient [42].

\section{Personalised management: partnership in care}

Personalised management emphasises and relies on the central role of the patient: patients have to become specialists of their own lives. In recent decades, the prevalence of chronic conditions has called for new approaches of handling patient care. Shared decision-making and the development of self-management tools are examples of such patient-centric healthcare processes. Shared decision-making has been defined as "an approach where clinicians and patients share the best available evidence when faced with the task of making decisions, and where patients are supported to consider options, to achieve informed preferences" [43]. According the World Health Organization, partnership in care is defined as "a collaborative relationship between two or more parties based on trust, equality and mutual understanding for achievement of a specified goal" [44]. Such partnership encompasses absolute interdependence, mutual accountability, collaborative relationship, equal participation in decision-making and shared power [44].

An informed and activated patient and a prepared practice team are crucial building blocks to realise this partnership. The healthcare professional has to take into account that individual health behaviours are created in a complex web of social, genetic, relational, individual and biological influences [45] and that people have different personalities, resilience and resources that influence their adaptation to illness [33].

As developments in healthcare and society are pushing towards a greater autonomy for the individual, health and care are increasingly put into the hands of the patient, thereby emphasising a greater individual autonomy. Expert patients are those who take responsibility for the day-to-day decisions about their 


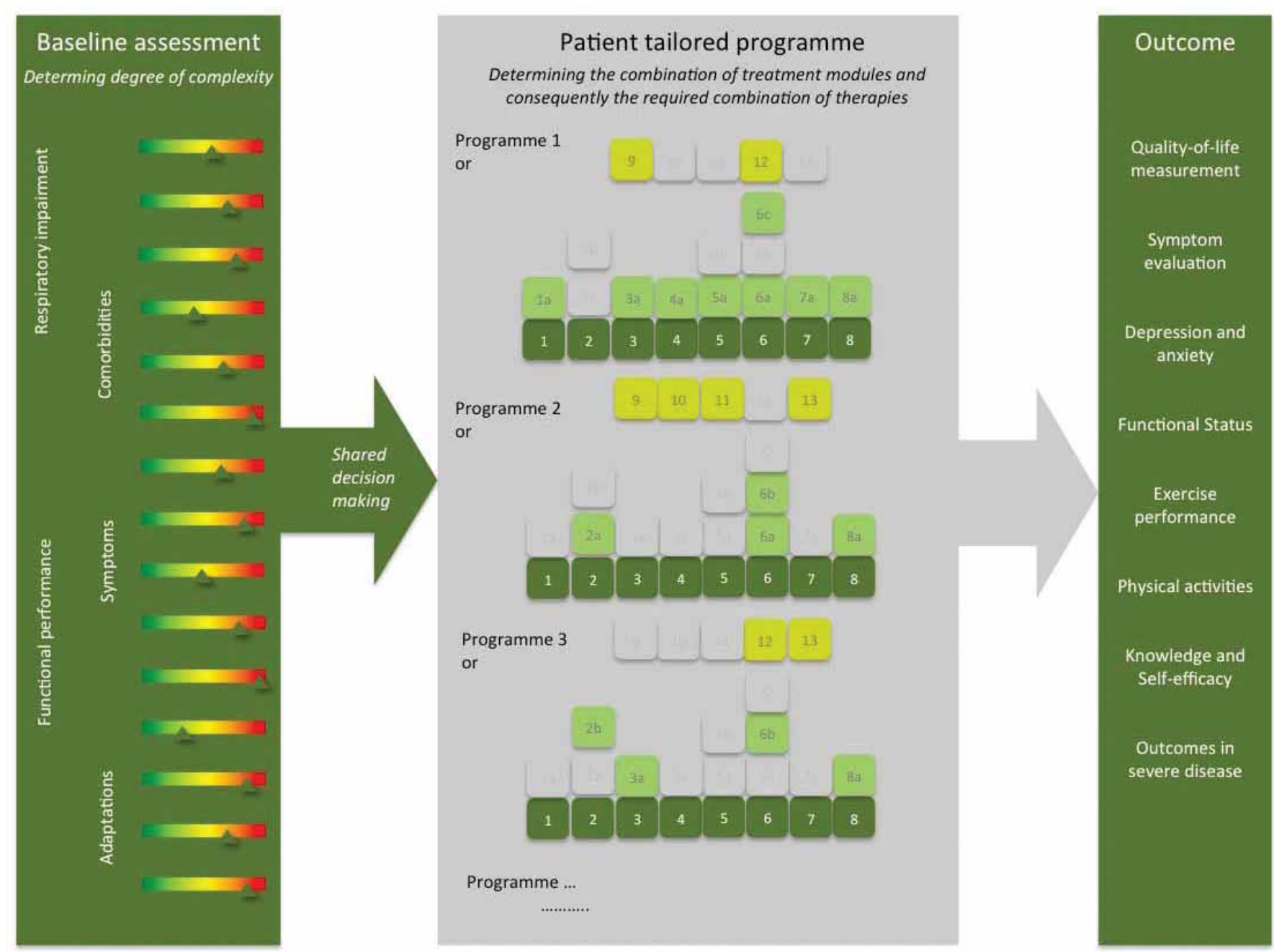

FIGURE 2 The process of a tailored programme including the three core processes (baseline assessment, tailored treatment and outcome assessment). Reproduced from [41] with permission.

health, and who work with healthcare providers as collaborators and partners to produce the best possible health given the resources at hand. Expert patients, especially those with chronic disorders such as COPD have been said to be producers of health, as well as consumers of healthcare [46]. Under all these circumstances, quality of care and respect for the patient's preferences and values need to be guaranteed.

It is within this context that personalised medicine can be seen as a means to strengthen individual autonomy while simultaneously putting the patient at the centre of the healthcare process. A greater individual autonomy requires provision of personal information to patients about their health and disease evolution. To further actively involve patients in this individualised care process and to increase the sense of responsibility for their own health, patients need access to their medical records and patients are encouraged to control and manage their own records and activities as well as possible, and should be able to evaluate the care process. When pulmonary rehabilitation aims to achieve the maximum level of independence and functioning in the community, providing skills to the patient to realise this partnership will become crucial. Ultimately, these specialists of their own lives can be embedded in an expert network. Indeed, health and disease management will become a consumer-driven business [47]. Creative new forms of engagement with these active participants in healthcare will become a major source of value tapped by the healthcare revolution [26].

\section{Conclusions}

Personalised or individualised medicine will fundamentally change the ways therapies are being developed and will explore how genetic, environmental and lifestyle factors interact in our bodies to foster health or develop disease. Besides biological variability, individuals differ greatly in terms of how disease affects their 
lives. Pulmonary rehabilitation offers an integrated approach for personalised management of patients with COPD, based on thorough assessment of treatable traits. Partnership in care will become crucial to maintain long-term adherence of patients to health-enhancing behaviours.

Conflict of interest: E.F.M. Wouters is a board member for Nycomed and Boehringer Ingelheim. He has received grants from AstraZeneca and GSK, and has received speakers' fees from AstraZeneca, GlaxoSmithKline, Novartis and Chiesi.

\section{References}

Speizer FE, Ware JH. Exploring different phenotypes of COPD. N Engl J Med 2015; 373: 185-186.

2 Global Initiative for Chronic Obstructive Lung Disease (GOLD). Global Strategy for the Diagnosis, Management and Prevention of COPD - 2016. http://goldcopd.org/global-strategy-diagnosis-management-prevention-copd-2016/

3 Eisner MD, Anthonisen N, Coultas D, et al. An official American Thoracic Society public policy statement: novel risk factors and the global burden of chronic obstructive pulmonary disease. Am J Respir Crit Care Med 2010; 182: 693-718.

$4 \quad$ Fletcher C, Peto R. The natural history of chronic airflow obstruction. Br Med J 1977; 1: 1645-1648.

5 Vanfleteren LE, Spruit MA, Groenen M, et al. Clusters of comorbidities based on validated objective measurements and systemic inflammation in patients with chronic obstructive pulmonary disease. Am J Respir Crit Care Med 2013; 187: 728-735.

6 Vanfleteren L, Spruit MA, Wouters EFM, et al. Management of chronic obstructive pulmonary disease beyond the lungs. Lancet Respir Med 2016; 4: 911-924.

7 Miller J, Edwards LD, Agusti A, et al. Comorbidity, systemic inflammation and outcomes in the ECLIPSE cohort. Respir Med 2013; 107: 1376-1384.

8 Agusti A, Bel E, Thomas M, et al. Treatable traits: toward precision medicine of chronic airway diseases. Eur Respir J 2016; 47: 410-419.

9 Hodgkin JE, Farrell MJ, Gibson SR, et al. American Thoracic Society. Medical Section of the American Lung Association. Pulmonary rehabilitation. Am Rev Respir Dis 1981; 124: 663-666.

10 Plsek PE, Wilson T. Complexity, leadership, and management in healthcare organisations. BMJ 2001; 323: 746-749.

11 Simpkin AL, Schwartzstein RM. Tolerating uncertainty - the next medical revolution? N Engl J Med 2016; 375: 1713-1715.

12 Bean R. Sir William Osler: Aphorisms from His Bedside Teachings and Writings. New York, Henry Schuman, 1950.

13 Annegarn J, Meijer K, Passos VL, et al. Problematic activities of daily life are weakly associated with clinical characteristics in COPD. J Am Med Dir Assoc 2012; 13: 284-290.

14 Vaes AW, Wouters EFM, Franssen FME, et al. Task-related oxygen uptake during domestic activities of daily life in patients with COPD and healthy elderly subjects. Chest 2011; 140: 970-979.

15 Stoilkova A, Janssen DJ, Franssen FM, et al. Coping styles in patients with COPD before and after pulmonary rehabilitation. Respir Med 2013; 107: 825-833.

16 Papava I, Oancea C, Enatescu VR, et al. The impact of coping on the somatic and mental status of patients with COPD: a cross-sectional study. Int J Chron Obstruct Pulmon Dis 2016; 11: 1343-1351.

17 Fraser DD, Kee CC, Minick P. Living with chronic obstructive pulmonary disease: insiders' perspectives. $J$ Adv Nurs 2006; 55: 550-558.

18 Wouters EF, Augustin IM. COPD health-care delivery: a holistic and dynamic approach is needed. Lancet Respir Med 2016; 4: e30-e31.

19 Kaljouw M, van Vliet K. Moving Towards New Health Care and New Health Care Professions: the Contours. 2016. https://english.zorginstituutnederland.nl/publications/reports/2015/04/10/moving-towards-new-health-careand-new-health-care-professions-the-contours

20 Huber M, Knottnerus JA, Green L, et al. How should we define health? BMJ 2011; 343: d4163.

21 PHG Foundation. Many Names for One Concept or Many Concepts in One Name? www.phgfoundation.org/file/ 13380 Date last accessed: June 6, 2017.

22 National Cancer Institute. Personalized Medicine. www.cancer.gov/publications/dictionaries/cancer-terms? cdrid=561717 Date last accessed: June 6, 2017.

23 President's Council of Advisors on Science and Technology. Priorities for Personalized Medicine. September 2008. http://oncotherapy.us/pdf/PM.Priorities.pdf

24 Scholz N. Personalised Medicine: the Right Treatment for the Right Person at the Right Time. 2015. www.europarl. europa.eu/RegData/etudes/BRIE/2015/569009/EPRS_BRI\%282015\%29569009_EN.pdf Date last accessed: June 6, 2017.

25 Mancinelli L, Cronin M, Sadée W. Pharmacogenomics: the promise of personalized medicine. AAPS PharmSci 2000; 2: E4.

26 Hood L, Flores M. A personal view on systems medicine and the emergence of proactive P4 medicine: predictive, preventive, personalized and participatory. N Biotechnol 2012; 29: 613-624.

27 Jameson JL, Longo DL. Precision medicine - personalized, problematic, and promising. N Engl J Med 2015; 372: 2229-2234.

28 National Research Council. Toward Precision Medicine: Building a Knowledge Network for Biomedical Research and a New Taxonomy of Disease. Washington, DC, The National Academies Press, 2011.

29 Xie H, Frueh F. Pharmacogenomics steps toward personalized medicine. Pers Med 2005; 2: 325-337.

30 Topol EJ. Individualized medicine from prewomb to tomb. Cell 2014; 157: 241-253.

31 FORUM Academy of Medical Sciences. Stratified, Personalised or P4 Medicine: a New Direction for Placing the Patient at the Centre of Healthcare and Health Education. 2015 https://acmedsci.ac.uk/viewFile/ 564091e564072d564041.pdf Date last accessed: June 6, 2017.

32 Wilson T, Holt T, Greenhalgh T. Complexity science: complexity and clinical care. BMJ 2001; 323: 685-688.

33 Ziegelstein RC. Personomics. JAMA Intern Med 2015; 175: 888-889. 
34 Han MK, Agusti A, Calverley PM, et al. Chronic obstructive pulmonary disease phenotypes: the future of COPD. Am J Respir Crit Care Med 2010; 182: 598-604

35 Pinto LM, Alghamdi M, Benedetti A, et al. Derivation and validation of clinical phenotypes for COPD: a systematic review. Respir Res 2015; 16: 50.

36 Bourbeau J, Pinto LM, Benedetti A. Phenotyping of COPD: challenges and next steps. Lancet Respir Med 2014; 2: $172-174$

37 Kosteli MC, Heneghan NR, Roskell C, et al. Barriers and enablers of physical activity engagement for patients with COPD in primary care. Int J Chron Obstruct Pulmon Dis 2017; 12: 1019-1031.

38 Fishman AP. Pulmonary rehabilitation research. Am J Respir Crit Care Med 1994; 149: 825-833.

39 Spruit MA, Singh SJ, Garvey C, et al. An official American Thoracic Society/European Respiratory Society statement: key concepts and advances in pulmonary rehabilitation. Am J Respir Crit Care Med 2013; 188: e13-e64.

40 Lusuardi M, Ambrosino N, Donner C. Definition and rationale for pulmonary rehabilitation. In: Donner C, Ambrosino N, Goldstein R, eds. Pulmonary Rehabilitation. London, Arnold, 2005; pp. 3-8.

41 Augustin I, Spruit M, Franssen F, et al. Understanding complexity of chronic non-communicable diseases: an integrated approach for personalized management of patients with COPD. Clin Res Pulmonol 2015; 3: 1034-1042.

42 Spruit MA, Augustin IM, Vanfleteren LE, et al. Differential response to pulmonary rehabilitation in COPD: multidimensional profiling. Eur Respir J 2015; 46: 1625-1635.

43 Elwyn G, Laitner S, Coulter A, et al. Implementing shared decision making in the NHS. BMJ 2010; 341: c5146.

44 World Health Organization (WHO). Building a Working Definition of Partnership. 2009. www.who.int/ patientsafety/implementation/apps/resources/defining_partnerships-apps.pdf Date last accessed: Nov 6, 2017.

45 Bauman AE, Fardy HJ, Harris PG. Getting it right: why bother with patient-centred care? Med J Aust 2003; 179: 253-256.

46 Lorig K. Partnerships between expert patients and physicians. Lancet 2002; 359: 814-815.

47 Knowledge@Wharton. Precision Medicine: New Paradigms, Risks and Opportunities. 2016 http://d1c25a6gwz7q5e. cloudfront.net/reports/2016-08-22-Precision-Medicine-New-Paradigms-Risks-and-Opportunities.pdf Date last accessed: June 6, 2017 